\title{
STEREOLOGY OF NANO-MATERIALS
}

\section{Tomasz Wejrzanowski, MaŁgorzata Lewandowska And KRZYszTof J. KURZYDŁOWSKI}

Warsaw University of Technology Faculty of Materials Science and Engineering, Woloska 141, 02-507 Warsaw, Poland

e-mail: twejrzanowski@inmat.pw.edu.pl,malew@inmat.pw.edu.pl,kjk@inmat.pw.edu.pl

(Accepted October 14, 2009)

\begin{abstract}
Nano-structured materials attract a growing attention due to their superior mechanical and physical properties. Such properties are inherently related to the unique structure which is controlled at the nano-scale. In the early stage of their development, the structural characterization of nano-materials was limited to the average grain/particle size. However, nano-grains/particles form stochastic populations with diverse size and shape. The recent experimental and theoretical results show that in addition to the average size, size diversity of grains/particles has a significant effect on the properties of nano-materials. This rationalizes more advanced description of the structures of nano-materials. Advanced microscopic methods are now available to image microstructure of nano-materials, e.g., HRSTEM and AFM. The ultra-high resolution images can be efficiently processed to obtain quantitative description of the nano-grains/particles. Examples are shown how such a description can be used for optimizing the microstructures of modern engineering nano-materials.
\end{abstract}

Keywords: image analysis, nanomaterials, nanometals, quantitative description, stereology.

\section{INTRODUCTION}

Nano-materials attract a growing attention due to their superior mechanical and physical properties. Such properties are inherently related to their structure which is controlled at the nano-scale, in particular to the effect of nano-sized grains (nano-polycrystalline materials) and particles (nanocomposites). The growing interest in nano-materials has brought about the development of new fabrication routes, which allow production of relatively large quantities of nano-artefacts.

Recent development in nano-materials intensified the need for a better understanding of structureproperties relationship in engineering materials, in particular at the nano-scale range. An example of such a relationship is shown in Fig. 1, which provides data on the strength of aluminium alloys as a function of the grain size, $\left(d_{2}\right.$, Widlicki, 2008). It can be noted that the strength can be improved by a factor of 2 to 5 , if the grain size of the alloy in question is reduced to nanometres.

In the early stage of the development of nanomaterials, their structural characterization was limited to the average grain/particle size. However, nanograins/particles form stochastic populations with diverse size and shape.

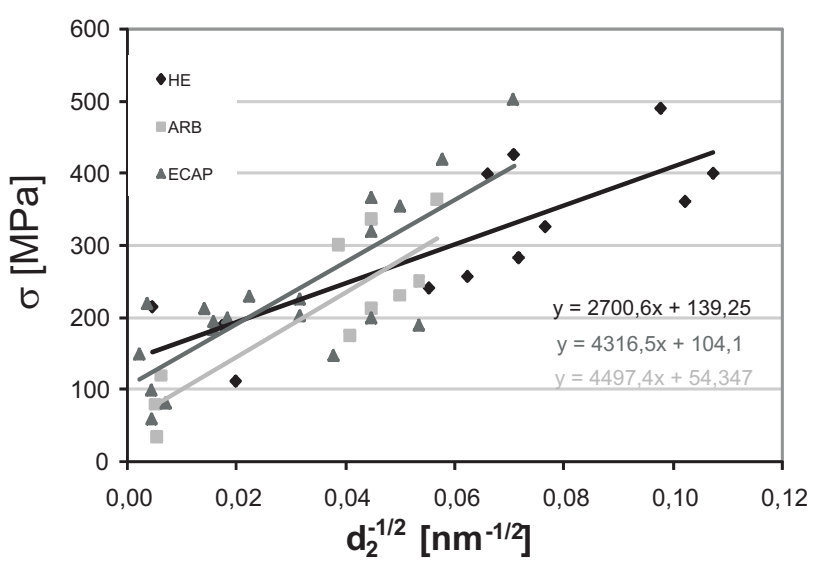

Fig. 1. Effect of grain size on the strength of aluminium alloys subjected to grain refinement via three techniques of Severe Plastid Deformation: Hydrostatic extrusion (HE), Accumulative Rolling/Bending (ARB) and Equal Channel Angular Pressing (ECAP).

The recent experimental and theoretical results (Dobosz, 2009) show that in addition to the average size, size diversity of grains/particles (usually defined as variation coefficient, $\mathrm{CV}\left(d_{2}\right)$ ) has a significant effect on the properties of nano-materials which is clearly visible in Fig. 2. This rationalizes more advanced description of the structures of nano-materials, which can be quantified using the methods of modern image analysis and stereology.

In the case of composites strengthened with nano-particles, the crucial point is their homogenous spatial dispersion. Quantification methods for 
measuring dispersion of particles in a matrix have been known for years (Hanisch and Stoyan, 1981; Wojnar and Majorek, 1994). However, application of these methods to nano-composites requires sub-nano resolution imaging techniques and special methods for sample preparation.

The paper reviews the possibilities of nanoscale imaging and quantitative description of nanostructures. It will be shown that the ultra-high resolution images can be efficiently processed to obtain quantitative description of the nanograins/particles. Examples will be given how such a description can be used for optimizing the microstructures of modern engineering nanomaterials.

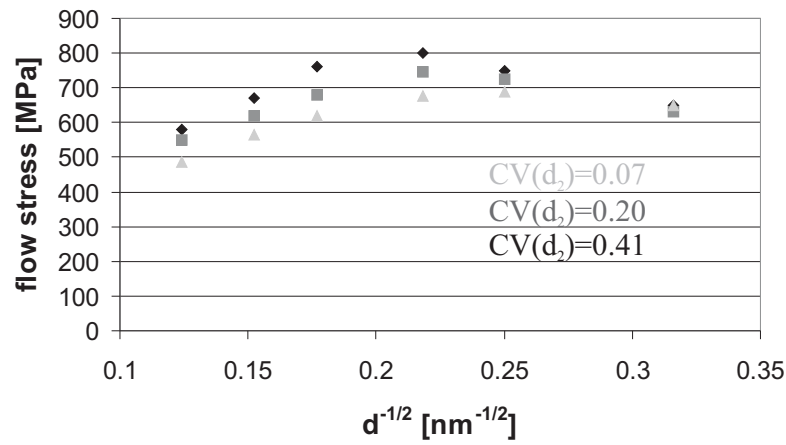

Fig. 2. The effect of grain size homogeneity described by variation coefficient, $C V\left(d_{2}\right)$, on the flow stress of nano-metals.

\section{IMAGING TECHNIQUES AND SAMPLE PREPARATION}

The task of sizing nano-objects is challenging by itself as efficient procedures for providing quantitative description of nano-sized systems are becoming available only recently. This is due to the fact that imaging of nanometre size objects requires high magnifications typically in the range of $10^{5}$ $10^{6}$, which is offered only by electron and more recently atomic force microscopes. Recent advances in electron/atomic force microscopic techniques made them more widely accessible and much more user friendly bringing about a major progress in the technology of nano-materials. In particular, one should acknowledge fast development in microscopic methods based on electron scanning probes, power of which is demonstrated with the examples given in further text.

\section{Sample preparation}

Modern high resolution microscopy by definition requires careful techniques for sample preparation. The general rule is that the higher magnification, the smaller samples can be examined. Also, these samples need to be cut-out with the growing precision out of bigger artefacts with minimum damage to their microstructure. The surface treatment after cutting should also be more sophisticated. This was also possible in the past using conventional techniques, e.g., electro-polishing.
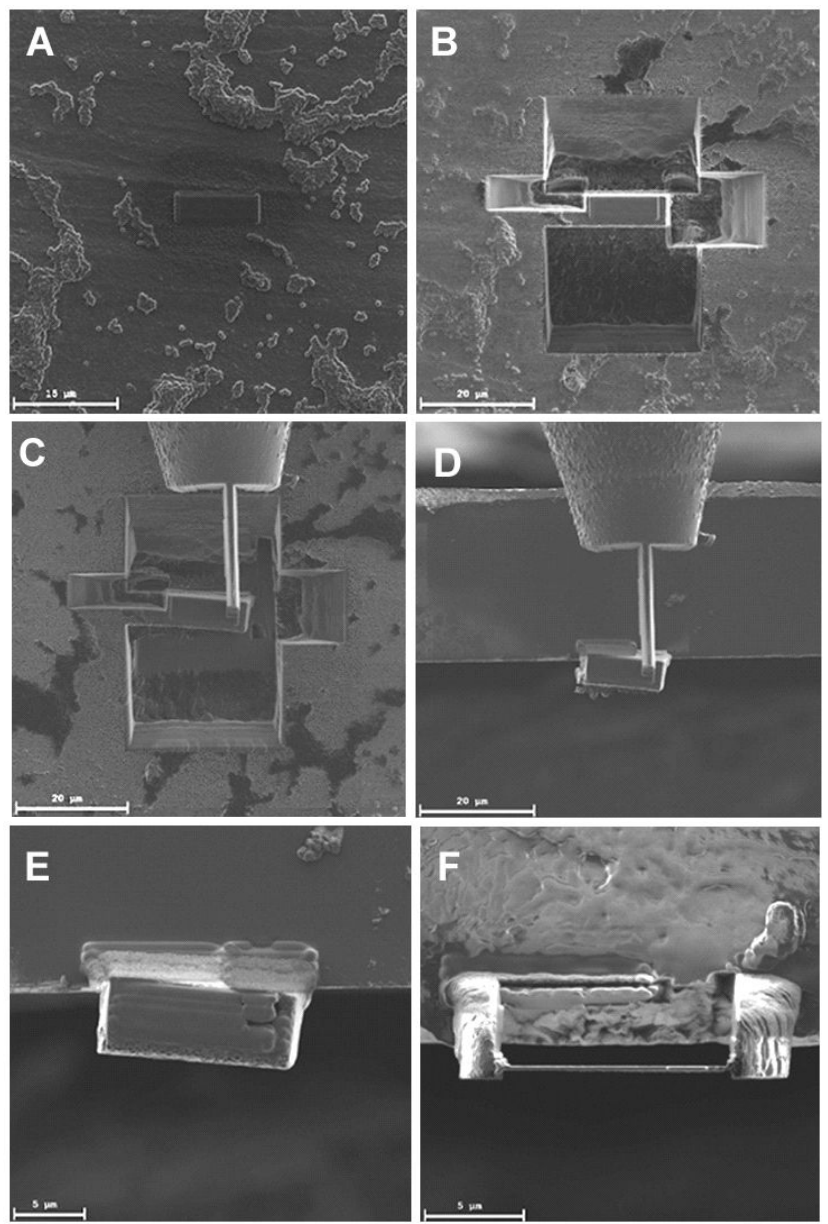

Fig. 3. Series of images illustrating capacity of Focused Ion Beam technique as applied to cutting small specimens out of a bigger artefact: A: area of interest; $B$ : the area of interest separated from the "matrix"; $C$ : a needle attached to the small sample; $D$ : the small sample taken out of the artefact; E: the general image of the small sample; $F$ : the structure of the sample.

However, these techniques provided efficient solutions only in the case of relatively homogenous and conductive materials, in particular metals and alloys. They were ineffective in the case of multiphase materials such as polymer based composites, which consist of phases differing significantly in their 
properties (hard particles embedded in the soft matrix). These difficulties have been recently overcome by a wider use of Focused Ion Beam techniques, FIB, which have been used in the past in examining of electronic devices. Recently, they have been adopted in the studies of nano-designed materials. The consecutive stages in sample preparation by FIB are exemplified by the series of images in Fig. 3. The advantage of this technique is the possibility of preparing thin specimens made of complex multi-phase materials (polymer matrix composite), which can be cut out from a selected place (e.g., interface or surface layer).

\section{Multi-scale imaging}

Modern nano-engineered materials usually reveal complex structure in different length scales: from nano- to micro-metres. This can be exemplified by the micrographs shown in Fig. 4, which reveals structure of polymeric matrix nano-composites used in biomedical (dental) applications. Such composites contain a whole range of ceramic particles, added to the matrix to obtain a desirable combination of properties. Size of some of them is in the range of micrometers to assure good wear resistance in the human mouth environment. On the other hand, nano-sized silica particles improve properties of the polymeric matrix. A comprehensive imaging of the structure of such composites requires cascade type of examinations, carried out with increasing magnification from 10000 , to reveal the larger particles, down to 150000 , to view the nano-sized ones.

\section{Complementary imaging techniques - TEM and SEM}

Modern electron microscopes are becoming the major tools for revealing structure of nano-designed engineering materials. These microscopes, in general, can be used in transmission or scanning mode, with each mode providing complementary information about the size, shape and density of structural features.

Generally, scanning electron microscopy (SEM) is used to reveal the features emerging on the surface. To a considerable degree the images obtained in this way emulate classical optical ones, known from the textbooks on the physical metallurgy. A great advantage of SEM is that it provides information not only on surface topography but also on surface chemical composition. On the other hand, transmission electron microscopy (TEM) provides projected images of the structure, more widely used in biology. In general, TEM offers higher magnification and the ability to obtain image and diffraction pattern.

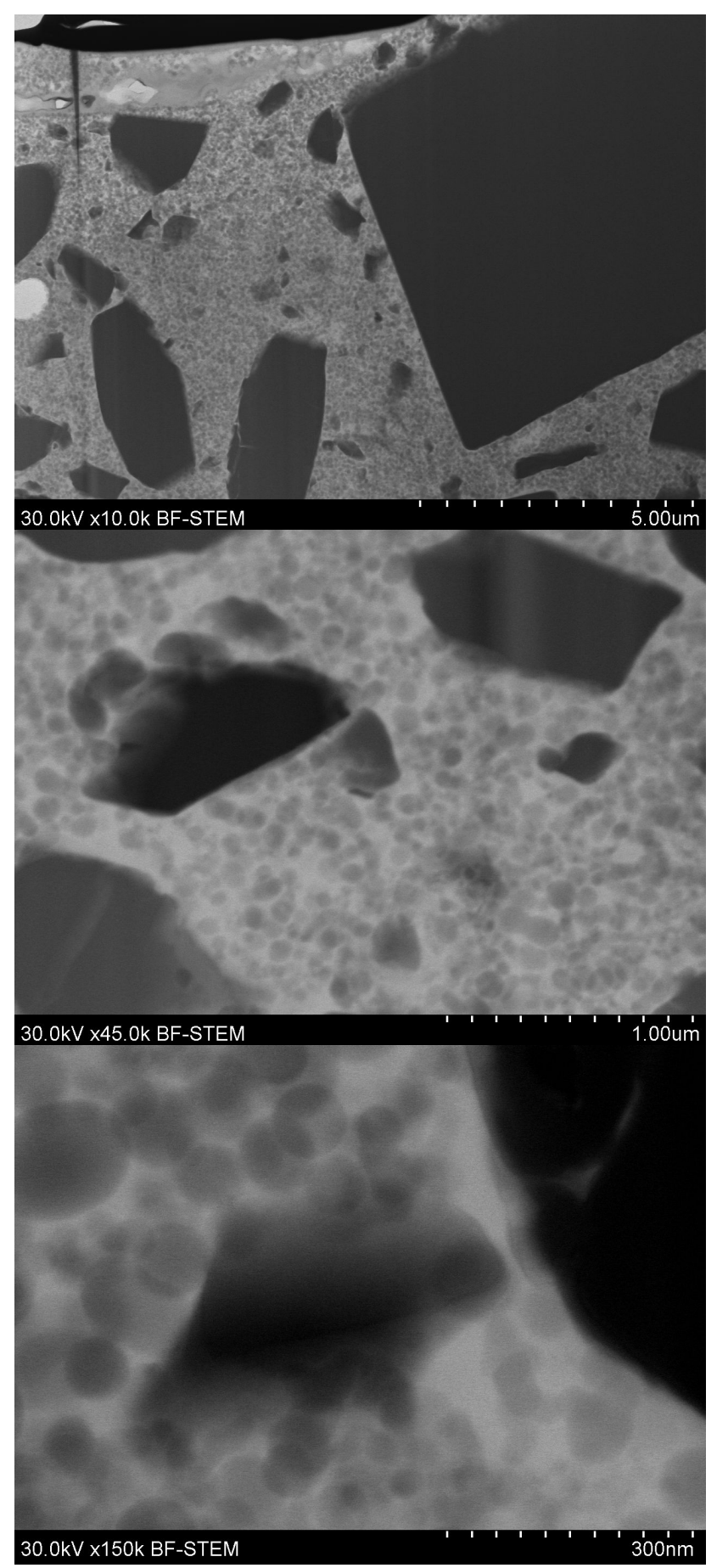

Fig. 4. Series of images illustrating microstructure of the composites used for dental application with progressive magnifications obtained with scanning transmission electron microscopy.

An important development in the field of nanostructure imaging over last years has come with the advances in the scanning-transmission devices (STEM), which can be used to obtain images of the same area using these two different modes and providing information on size/shape, chemistry and spatial distribution of nano-scale structure elements. 

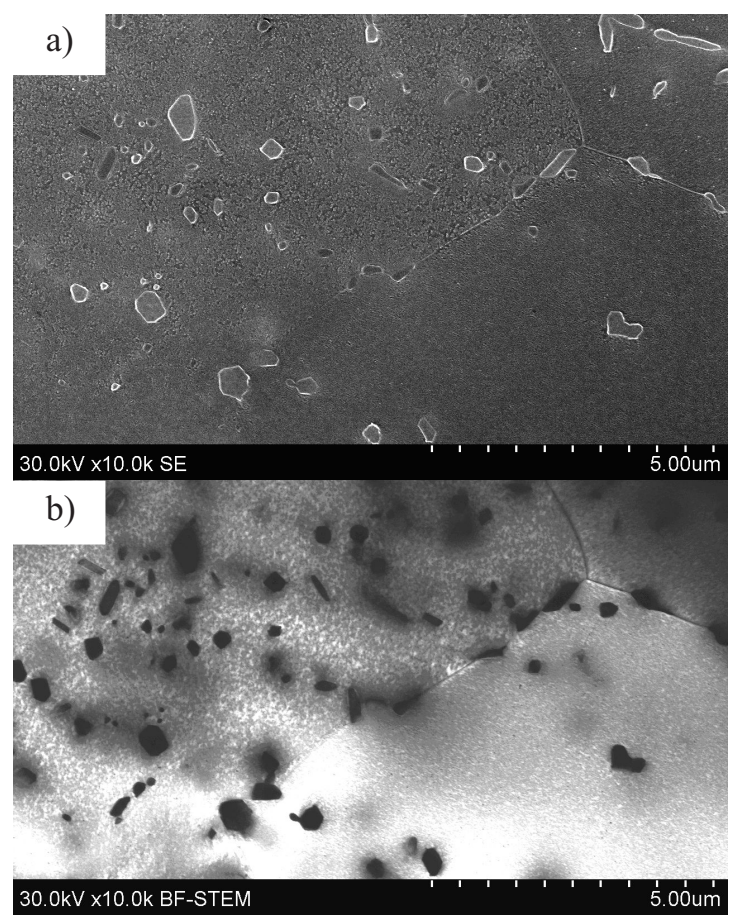

Fig. 5. Sub-micron carbide precipitates observed in: (a) secondary electrons (only carbides located on the specimen surface are visible) and (b) transmitted electrons (all carbides present in the specimen are revealed).

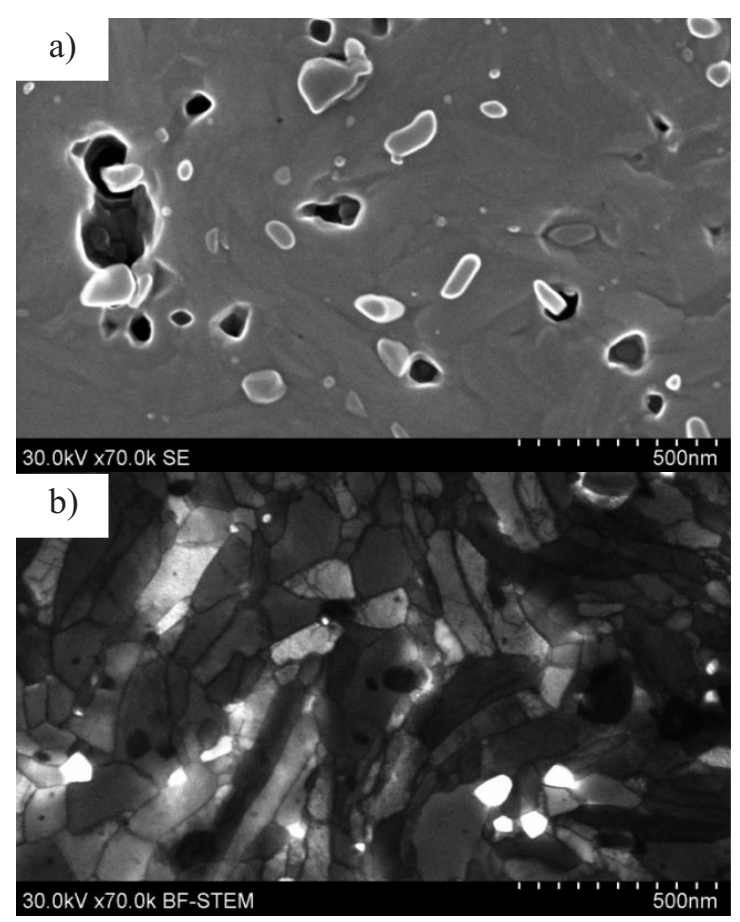

Fig. 6. Structure of Eurofer 97 steel $(\mathrm{Fe}-0.08 \%$ $\mathrm{C}-0.4 \% \mathrm{Mn}-9 \% \mathrm{Cr}-1.1 \% \mathrm{~W}-0.2 \% \mathrm{~V}-$ $0.14 \% \mathrm{Ta}-0.3 \% \mathrm{Y}_{2} \mathrm{O}_{3}$ ): (a) carbides and residual porosity revealed in secondary electron; (b) nanograin structure observed with transmission electrons.
In STEM, electron optics focus the beam into a narrow spot which is scanned over the sample (like in conventional scanning microscope) but electrons which passed through the specimen can also be detected as a transmission mode image. This offers the possibility to obtain complementary information from the same place of observation. Examples of image complementarities in various observation modes are given in Figs. 5 and 6.

The resolution of modern STEM microscopes can achieve $0.12 \mathrm{~nm}$ which is competitive with conventional TEM. With such an atomic resolution, images can be obtained with contrast being directly related to the atomic number (no need of interpretation by simulation). The further advantages of STEM include rastering, which is suitable for analysis techniques, e.g., EDX, EELS.

\section{D imaging}

Another breakthrough in the imaging of materials structures has come recently due to the advances in the X-ray tomography. Modern desk-top tomography unit are now available at acceptable price, which can be used to reveal structure of materials at nano-/microresolution. Images, obtained using this technique, are shown in Fig. 7. It should be noted that such images allow for a full 3-D reconstruction of the structure of interest.
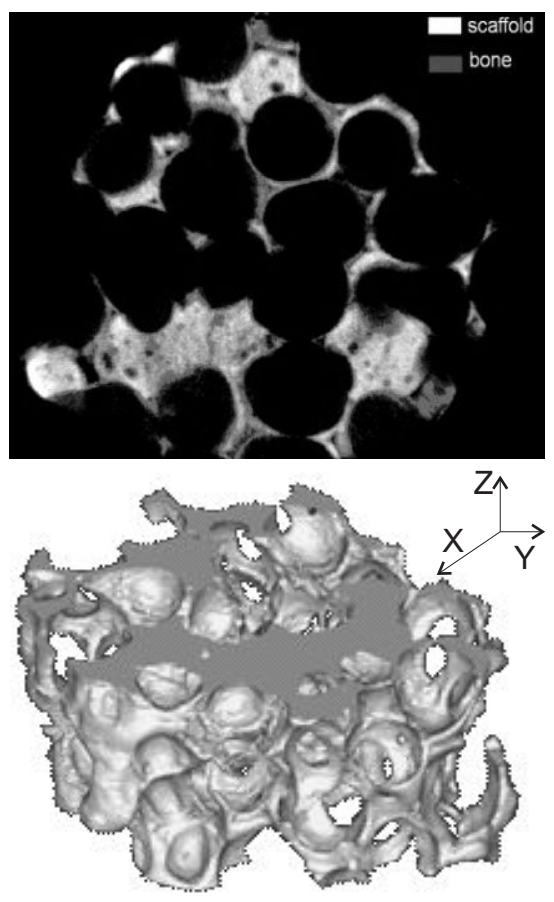

Fig. 7. Images of the porous material for biomedical applications (scaffolds used in tissue engineering) obtained using nano-tomography. 


\section{QUANTITATIVE DESCRIPTION}

Interfaces, grains and inter-phase boundaries are the major features of the nano-designed materials. Simple consideration show that the volume fraction of atoms located in grain boundaries (GBs) in nano-polycrystalline material may exceed $25 \%$. Also, nano-particles account for huge specific surface area of inter-phase boundaries in composite materials. This situation necessitates development of efficient procedures for measurements of density of interfaces in nano-materials.

\section{Quantitative description of grain boundaries}

Geometry of grain boundaries can be quantitatively described using principles of stereology and image analysis. From a stereological point of view, grain boundaries form an inter-connected set of curved 2-D objects. Total surface area of such objects in unit volume, $S_{V}$, can be estimated using relatively simple techniques explained in Fig. 8 (for more details see Kurzydlowski and Ralph, 1995). As one can see this technique is extremely simple in the case of the grain boundaries, which are oriented randomly in space. In the case of boundaries exhibiting a measurable degree of anisotropy (morphological texture), vertical sectioning method is required, introduced in Baddeley et al. (1986)).
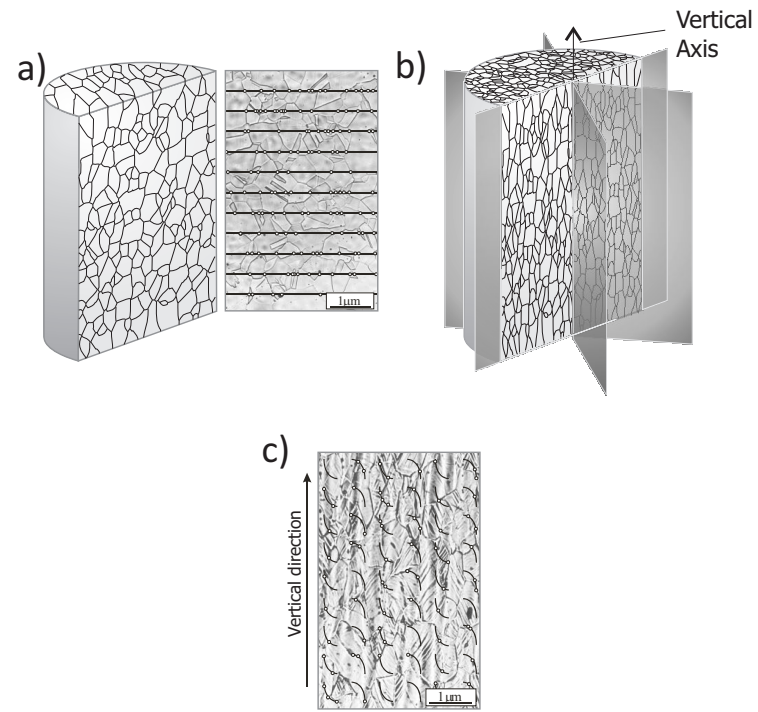

Fig. 8. Measurements required for un-biased estimate of the surface area of grain boundaries in unit volume of material: (a) isotropic geometry of grain boundaries; (b) vertical sectioning and (c) cycloids used in the anisotropic case.

The value of $S_{V}$ parameter can be estimated both in the case of truly 2-D boundaries and for the boundaries, which exhibit certain thickness, $t$. In the latter case, the product of $S_{V}$ and t provides an estimate of the volume fraction of "grain boundary" phase, $V_{V}$ :

$$
V_{V}=S_{V} \cdot t
$$

Stereological arguments show that surface of the grain boundaries in unit volume can also be used to estimate the mean intercept length, $\bar{l}$ which is used in pile-up model of grain boundary effect on flow stress of polycrystals (Armstrong, 1983). Geometrical probability theory shows that the mean intercept length of grains is proportional to $\left(S_{V}\right)^{-1}$ :

$$
\bar{l}=2 / S_{V} . \quad[\mathrm{m}]
$$

However, in the case of nano-crystalline material with grain boundaries of non-zero thickness, a correction factor is required and $\bar{l}$ is given by the following formula:

$$
\bar{l}=2 / S_{V}-t . \quad[\mathrm{m}]
$$

Again it should be emphasized that for anisotropic orientation of the grain boundaries, $S_{V}$ value needs to be estimated from images obtained on vertical sections.

The area of interfaces between particles and the matrix in dispersed-like structures depends on the particle size, but also it is related with the volume fraction of particles, $V_{V}$. In such a case, $S_{V}$ can be calculated from the following equation:

$$
S_{V}=4 V_{V} / \bar{l} . \quad\left[\mathrm{m}^{-1}\right]
$$

\section{Grain size and grain size homogeneity}

If the investigation of nano-materials is focused on grains rather than on grain boundaries, the most natural size parameter is grain volume, $\mathrm{V}$. The mean value of grain volume can be estimated by indirect methods (see, e.g., Ungar et al., 2002). Direct measurements can be based on one of the following procedures:

a) di-sector

b) point sampled intercepts

c) Saltykov reconstruction

The di-sector technique is explained in Fig. 9. In this case, grains are counted in a given, usually small, volume of the specimen which either are entirely within this volume or are cut only by half of the external surfaces. The density of grains can be subsequently obtained by dividing the number of counted grains, $\mathrm{N}$, by the studied volume of specimen, $V_{o}$ :

$$
N_{V}=N / V_{o} . \quad\left[\mathrm{m}^{-3}\right]
$$




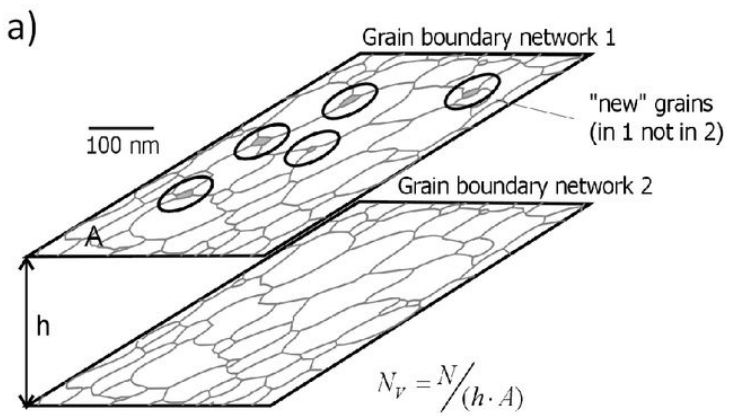

b)

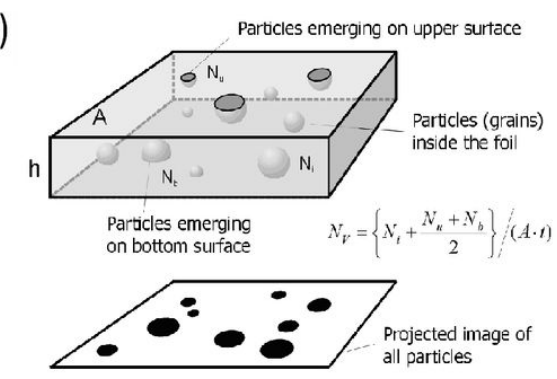

Fig. 9. Explanation of the di-sector measurements in the case of observations on sections (a) and projections $(b)$.

Again, the above given equation should be slightly corrected in the case of "thick grain boundaries". The correction factor is $\left(1-t \cdot S_{V}\right)$ :

$$
N_{V}=\left(1-t \cdot S_{V}\right) \cdot N / V_{o} \cdot\left[\mathrm{m}^{-3}\right]
$$

Once the $N_{V}$ value is known, $\bar{V}$ is calculated as $\left(N_{V}\right)^{-1}$ :

$$
\bar{V}=1 / N_{V} \cdot\left[\mathrm{m}^{3}\right]
$$

Saltykov reconstruction can be used to obtained full distribution function of the grain volume, $f(V)$. However, this method requires that the shape of grains needs to be specified. If this shape is fairly regular one may assume that geometry of grains can be approximated by Kelvin tetrahedron. This in turn leads to a system of algebraic equations which links the relative frequencies of grain sections/projections and grain volume - see Fig. 10.

Population of grains in polycrystals may differ not only in the mean value of their volume, $\bar{V}$, but also in terms of the width of the grain volume distribution function. (An example of such situation is given in Fig. 2). Such differences can be quantified using the ratio of standard deviation of grain volume, $\sigma_{V}$, and the mean value $\bar{V}-\sigma_{V} / \bar{V}$. For grains of the same volume $\sigma_{V} / \bar{V}=0$. Non-zero values of $\sigma_{V} / \bar{V}$ indicate diversity in the size of grains and the value of this ratio monotonically increases with increasing width of $f(V)$ function.

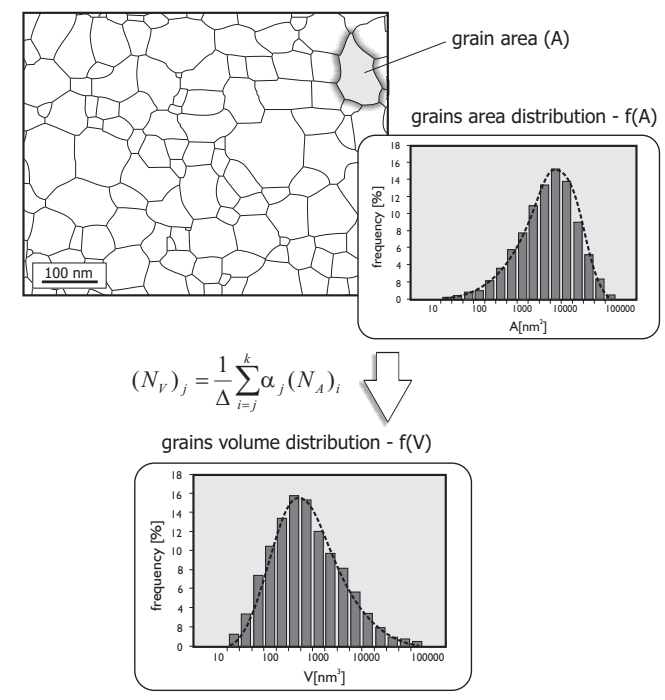

Fig. 10. Principles of Saltykov reconstruction.

Standard deviation of grain volume, $V$, can be directly calculated if Saltykov procedure is applied to estimate $f(V)$ function. Other method that can be used to estimate this parameter has been schematically explained in Fig. 11. As in the case of point sample intercepts, the estimates obtained in such a way bears a systematic error. This error is less important if the results are used simply to rank investigated structure in terms of grain size homogeneity.

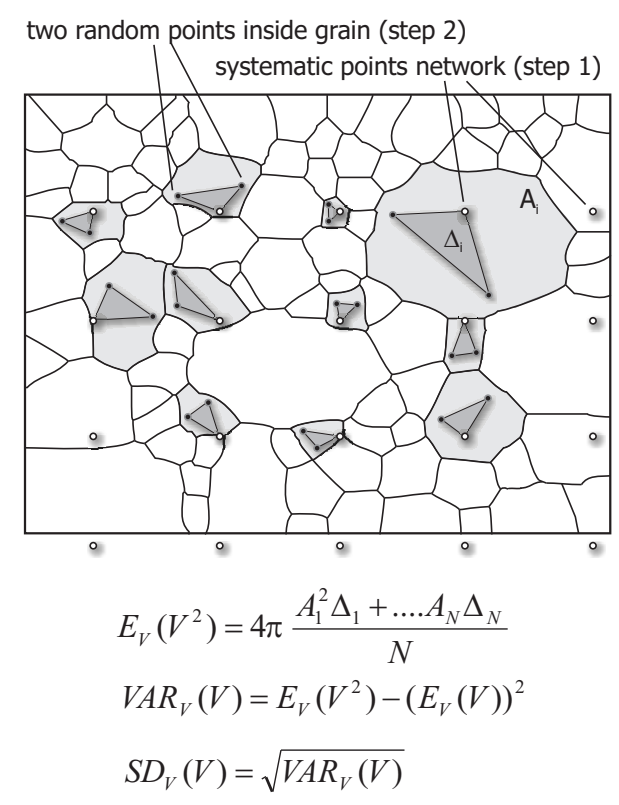

Fig. 11. Explanation of the method used to estimate the standard deviation of the grain volume.

\section{Spatial arrangement of particles}

Arrangement of particles within the volume of material can be investigated using the concept of covariance function, $c(r)$. This function defines probability that if a given randomly placed point hits such a particle, a point placed in distance $r$ also hits 
particle of that kind (including the particle hit by the first point itself). Some properties of covariance function are demonstrated in Fig. 12. The existence of maximum value of $c(r)$ for $r>0$ indicates that analyzed particles tend to be dispersed with some characteristic distance one with respect to others.

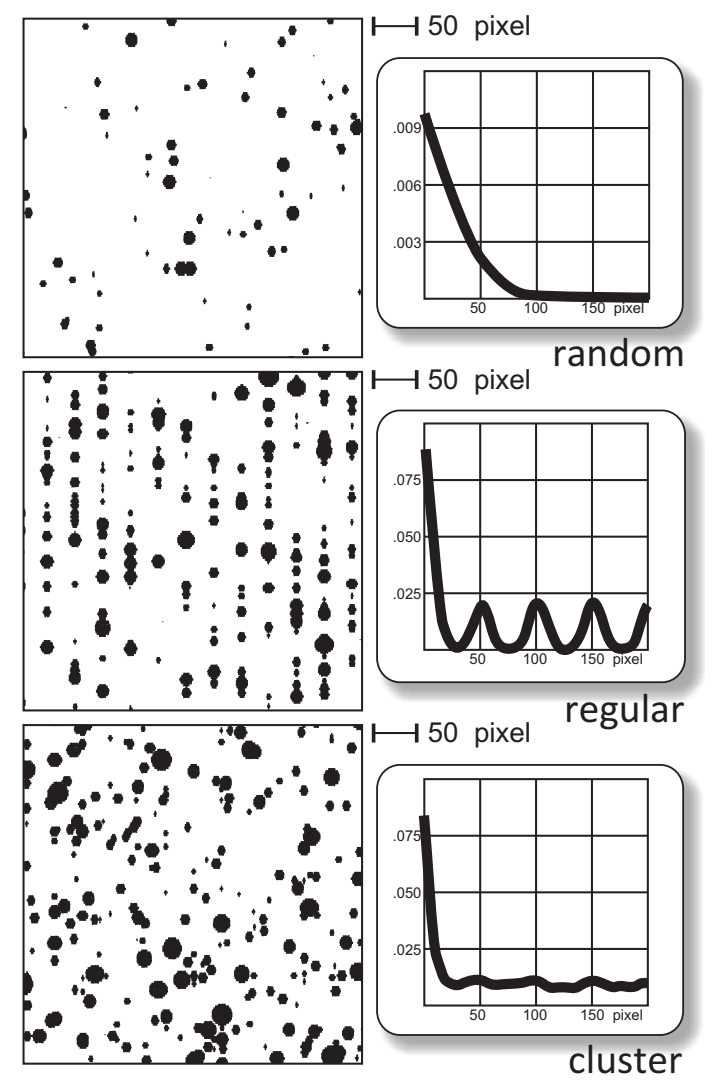

Fig. 12. Explanation of the properties of covariance function.

\section{APPLICATION TO NANOMATERIALS}

\section{Nano-polycrystalline materials}

Polycrystalline materials consist of a population of grains which usually differ in their size and shape. Depending on the average size of grains, described by the average value of the equivalent diameter or of the random intercept length, 3 following types of polycrystalline structures are distinguished by materials engineering community:

1. Micro-crystalline - grain size larger than $1 \mu \mathrm{m}$

2. Ultrafine grained - grain size in the range $0.1-1$ $\mu \mathrm{m}$

3. Nano-crystalline - grain size smaller than $0.1 \mu \mathrm{m}$
The above given classification has to a large degree a historical background, as the methods for grain size refinement in metals below $1 \mu \mathrm{m}$ became available only in the last 40 years and down to $100 \mathrm{~nm}$ only in the last 10 years. It is also rationalized by the variation in the properties of polycrystalline metals, which at relatively low temperatures are significantly strengthened by the grain boundaries (appearing in a larger density in lower grain sized materials). Quantitatively, the grain size strengthening is described by the Hall-Petch relationship, which predicts linear dependence of yields stress on the inverse square root of the average grain size. This implies a particularly significant increase in mechanical strength of metals with the grain size reduced to nano-range and gives rational to numerous efforts recently made to develop methods of grain size refinement.

There are a number of processing routes to obtain nano-polycrystalline materials, including consolidation of nano-powders. However, grain refinement via severe plastic deformation (SPD) seems to be particularly useful with regard to bulk metallic materials (Valiew et al., 2000). It has been demonstrated that SPD enables micro-grained aggregates to be transformed to nano-sized structures by accumulation and rearrangement/annihilation of the crystal lattice defects, primarily dislocations.

Obviously, the strengthening effect of plastic deformation has been known for years and is well described in textbooks of physical metallurgy in terms of defects accumulation, recovery and recrystallization. However, it has been recently shown that with the plastic deformation exceeding some critical value, deformed metals are transformed insitu (or after some post processing treatment) to aggregates of well developed nano-sized grains which render specific mechanical and physical properties. For such a transformation to take place, a large accumulation of crystal lattice defects is required, which is not achievable by the conventional methods of forming. In this context, special deformation methods have been developed, including Equal Channel Angular Pressing (Valiev and Langdon, 2006), High Pressure Torsion (Zhilayaev et al., 2002), Cyclic Extrusion Compression (Richert et al., 1999) and Multi Axial Forging (Cherukuri et al, 2005). More recently, Hydrostatic Extrusion, HE, has also been used to this end (Lewandowska et al, 2007; Lewandowska and Kurzydlowski, 2008).

It should be noted that SPD processing methods are far from being fully matured and are still under development. The challenge is in particular to optimize the process parameters, such as total plastic strain 
and strain rate for a given metallic "substrate". From this point of view it must be noted that the same deformation method applied to different metals results in refined microstructures which differ in grain size, size distribution, density of dislocation and character of grain boundaries (e.g., disorientation angle, content of twin boundaries). These differences are exemplified by the micrographs shown in Figs. 13 and 14, which illustrates microstructures induced by SPD processing in some engineering aluminium and iron alloys.

One may note that in all aluminium alloys, equiaxial grain structures have been developed, which differ significantly in average grain size. The largest grain size was obtained in commercial purity aluminium. In the case of aluminium alloys, the grain size is much smaller and a high density of dislocations appear inside the grains. For iron based materials, the observed structures are more diverse.
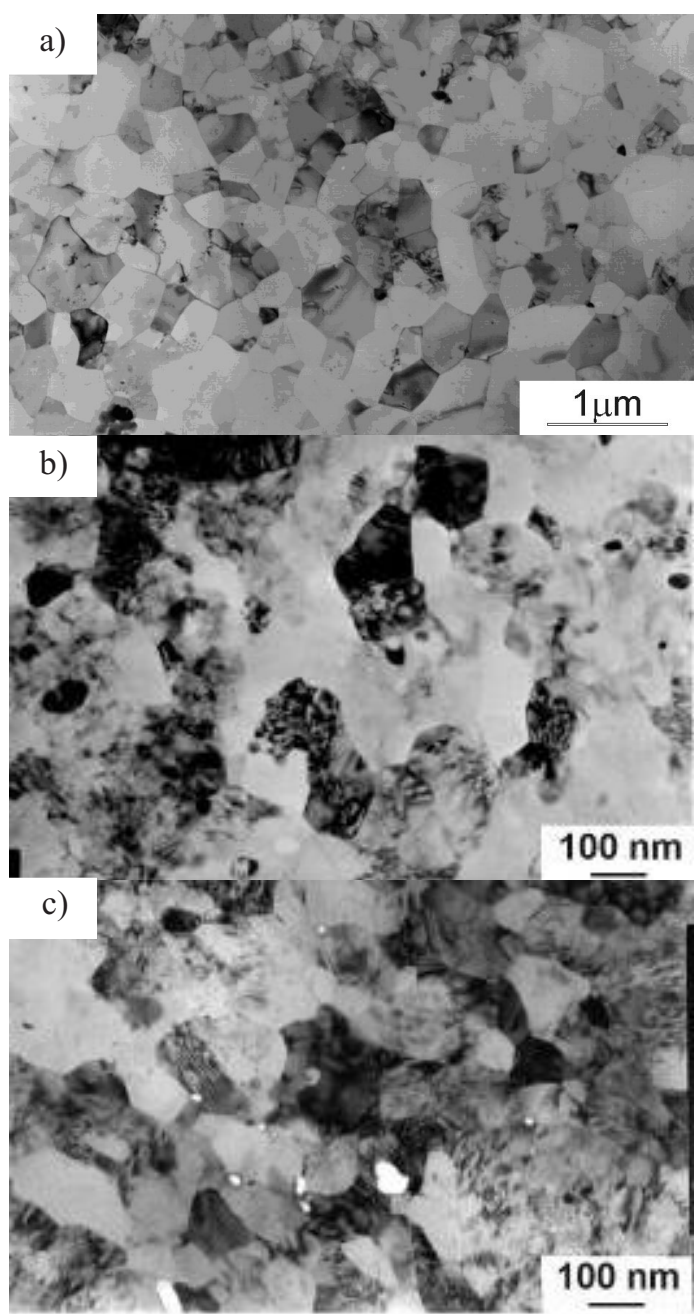

Fig. 13. Nanostructures induced by hydrostatic extrusion processing in aluminium based alloys: 1050 (a), 2017 (b), 7475 (c).
Well developed grain structure is observed in the case of commercial iron and ferritic steel, whereas stainless steel exhibits a high fraction of twin boundaries. Quantitative description of the grain size in such structures can be performed on the basis of TEM images. In this study, the size of individual grains was quantified using an equivalent diameter, $d_{2}$, which is defined as a diameter of a circle having the same surface area as a given grain. Grain size distribution functions for the structures presented in Figs. 13 and 14 are given in Fig. 15. The results show that nano-structures obtained via SPD processing reveal considerable non-homogeneity of grain size (grain size distribution functions are relatively wide). This calls for quantification of the grain size diversity, which can be carried out using coefficient of variation, $\mathrm{CV}\left(d_{2}\right)$, defined as a ratio of standard deviation to the mean value. The values of this coefficient and respective values of the average grain diameter for materials studied here are listed in Table 1.
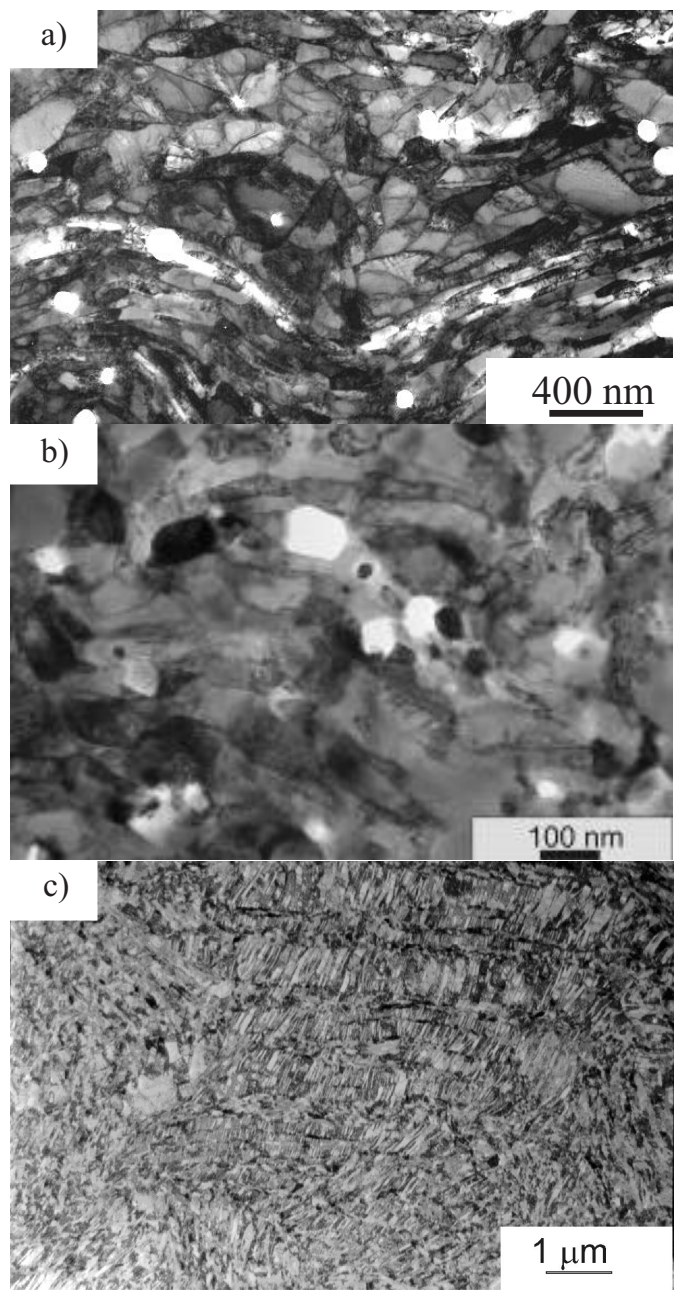

Fig. 14. Nanostructures induced by hydrostatic extrusion processing in iron based alloys: pure iron (a), ferritic EUROFER 97 steel (b) and austenitic stainless steel (c). 

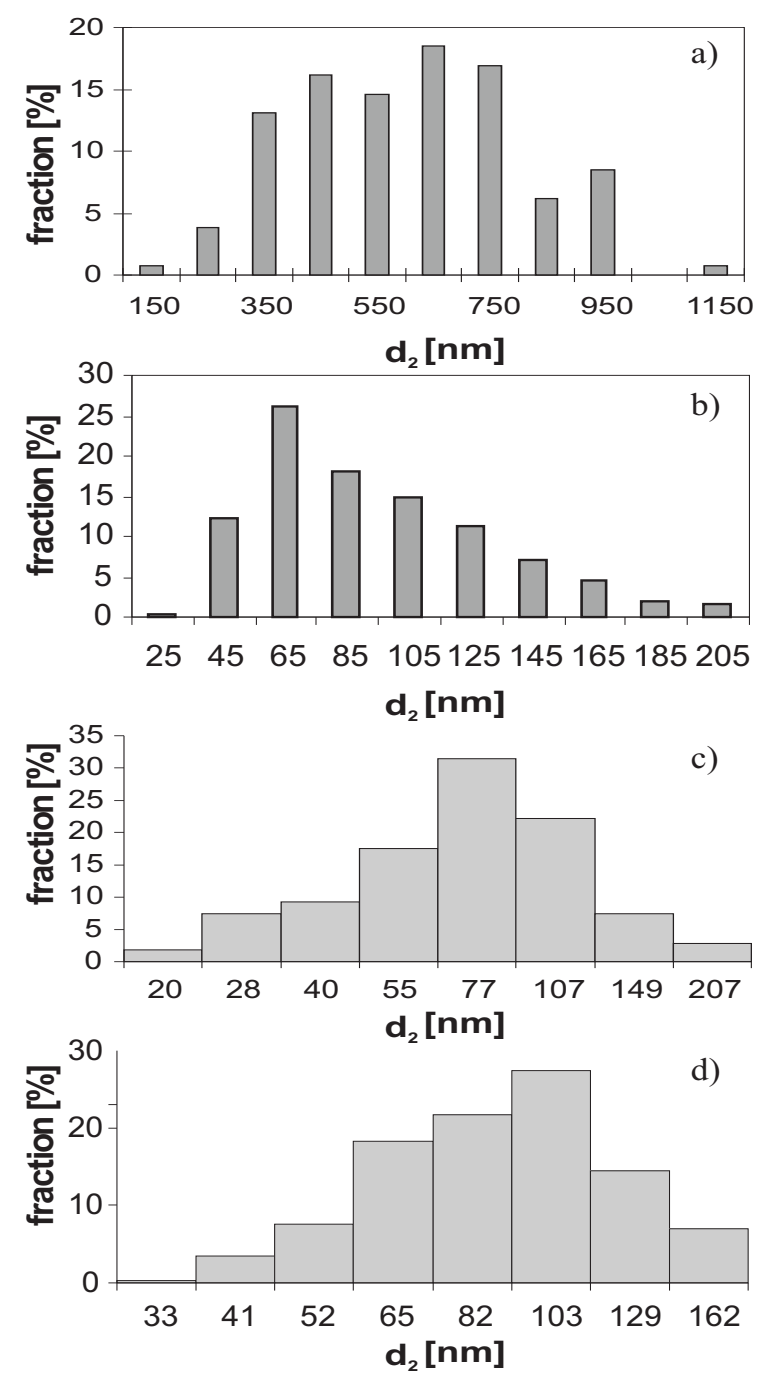

Fig. 15. Grain size distributions for aluminium based: 1050 (a), 2017 (b), and iron based: ferritic EUROFER 97 steel (c) and austenitic stainless steel (d).

Table 1. The results of measurements for the microstructures exemplified by micrographs shown in Figs. 13 and 14.

\begin{tabular}{lcccccc}
\hline \multirow{2}{*}{ Parameter } & \multicolumn{7}{c}{ Structure represented in Figure: } \\
\cline { 2 - 7 } & $13 \mathrm{a}$ & $13 \mathrm{~b}$ & $13 \mathrm{c}$ & $14 \mathrm{a}$ & $14 \mathrm{~b}$ & $14 \mathrm{c}$ \\
\hline$\left(d_{2}\right)[\mathrm{nm}]$ & 480 & 76 & 68 & 172 & 82 & 97 \\
$S D\left(d_{2}\right)[\mathrm{nm}]$ & 100.8 & 39.5 & 41.5 & 48.2 & 39.4 & 40.1 \\
$C V\left(d_{2}\right)$ & 0.21 & 0.52 & 0.61 & 0.28 & 0.48 & 0.42 \\
\hline
\end{tabular}

The data given in Table 1 indicate that the nanopolycrystalline and ultra-fine grained metals may differ in average grain size by a factor of 5 for the same processing parameters, depending on their chemical composition. This is due to the fact, that the final grain size is controlled by the rate of accumulation and annihilation/rearrangements of defects generated during SPD processing and concurrent thermally activated phenomena of recovery and recrystallization. As a result, in the case of deformation carried out at room temperature, the grain size is smaller for materials with high melting point (taking into account pure metals). Also, materials containing a large concentration of alloying elements, which in general decrease the rate of recovery and recrystallisation, reveal a lower grain size. These findings give indication of the possibility for controlling the final grain size of SPD processed metals by adjusting their chemical composition.

The data presented in Table 1 also show that the grain size diversity is higher with the smaller average grain size (see Fig. 16). As a result, metals with the average grain size below the "threshold" value of $100 \mathrm{~nm}$, contain considerable number of larger grains and should be considered as nano- submicroncomposites. In the drive for purely nano-sized metals, the diversity in their size must be controlled and kept low. On the other hand, there is experimental evidence that nano- sub-micron- composites may have better overall properties than purely nano-counterparts.

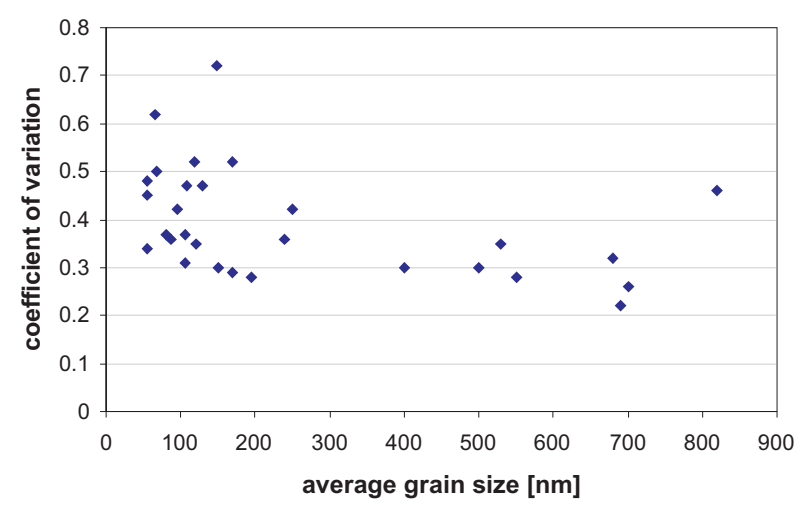

Fig. 16. Grain size diversity versus average grain size for various materials processed by hydrostatic extrusion.

In order to further elucidate the point made here on the importance of the grain size diversity in the case of nano- and sub-micron-metals, it can be noted that profoundly different grain structures may have the same average grain size, as exemplified by the micrographs in Fig. 17. These micrographs show microstructure of commercial aluminium processed by hydrostatic extrusion with different values of the applied strain. Both structures exhibit average grain size of $600 \mathrm{~nm}$, but one consists of grains fairly uniform in their size whereas in the other one can distinguish two populations of grains, which quite differ in the sizes.

Such diversity in the grain size as demonstrated in Fig. 16 has obviously significant impact on the 
mechanical properties of nano- sub-micron-metals. The samples with the microstructures presented in Fig. 17 exhibit more that $10 \%$ difference in the yield strength, with the ones revealing more homogenous grain size being more resistant to plastic deformation. This observation can be explained by finite element modelling, which provided quantitative estimates of the gain/loss due to the variation in the grain size homogeneity (Dobosz, 2009).

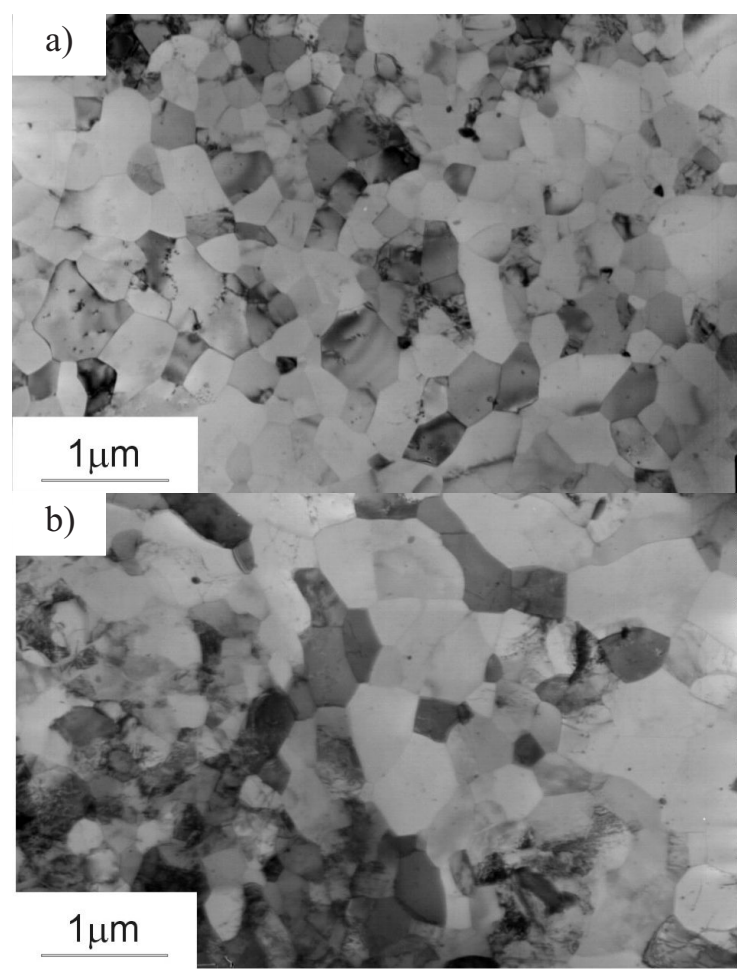

Fig. 17. Micrographs of commercial aluminium processed by a SPD method to two different final strains $3.8(a)$ and $2.7(b)$.

\section{Nano-composites}

Due to the recent development in technology of nano-powders, nano-sized particles are widely used as fillers/reinforcement phase in modern composite materials. The properties of such nano-composites are remarkably different in comparison to the composites with standard size fillers because of the high surfaceto-volume ratio of nano-particles. In particular, there is a strong improvement in the mechanical properties, such as modulus of elasticity and strength (Moszner and Saltz, 2001). Nano-composites reveal also higher hardness and abrasion resistance (Siejka-Kulczyk et al., 2009). They show remarkable dimensional/thermal stability and may exhibit special optical and electrical properties (Ryszkowska et al., 2007).

It has been demonstrated for a number of systems, e.g., for polymers modified with carbon nano-tubes, that addition of only a few percent of nano-sized particles can make a dramatic change in the properties and allow to obtain otherwise unachievable property combinations (Bauhofer and Kovacs, 2009). However, one should underline that all these new possibilities with regard to properties apply only in the case of nano-composites with homogenous spatial distribution of nano-sized fillers.

Uniform spatial distribution of the nano-particles in the composite matrix is a major technological challenge due to their huge surface energy and strong tendency for agglomeration. Once agglomerated, nano-particles lose beneficial effect on the properties of nano-composites and a variety of methods have been utilized to homogenize their spatial arrangement, in terms of regularity, clustering and randomness. In order to evaluate the efficiency of these methods, and to control nano-particles distribution, robust methods of microstructure imaging and quantitative characterization are required.

Fig. 18 illustrates two STEM images of dental nano-composites containing $40 \mathrm{vol} \%$ of nano-silica with the average particle size of $40 \mathrm{~nm}$. It can be noted that spatial arrangement of nano-particles in both microstructures is different. However, for unbiased analyses of these differences, the homogeneity of the nano-particles needs to be quantified.
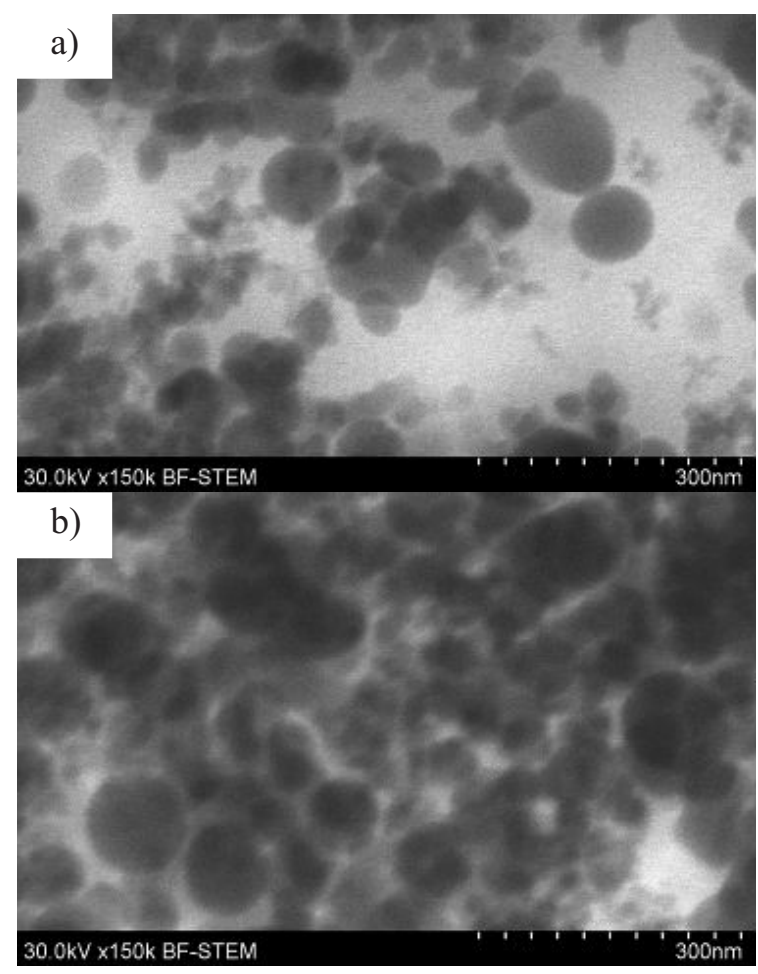

Fig. 18. Silica nano-particles in ceramic-polymer dental composites. 

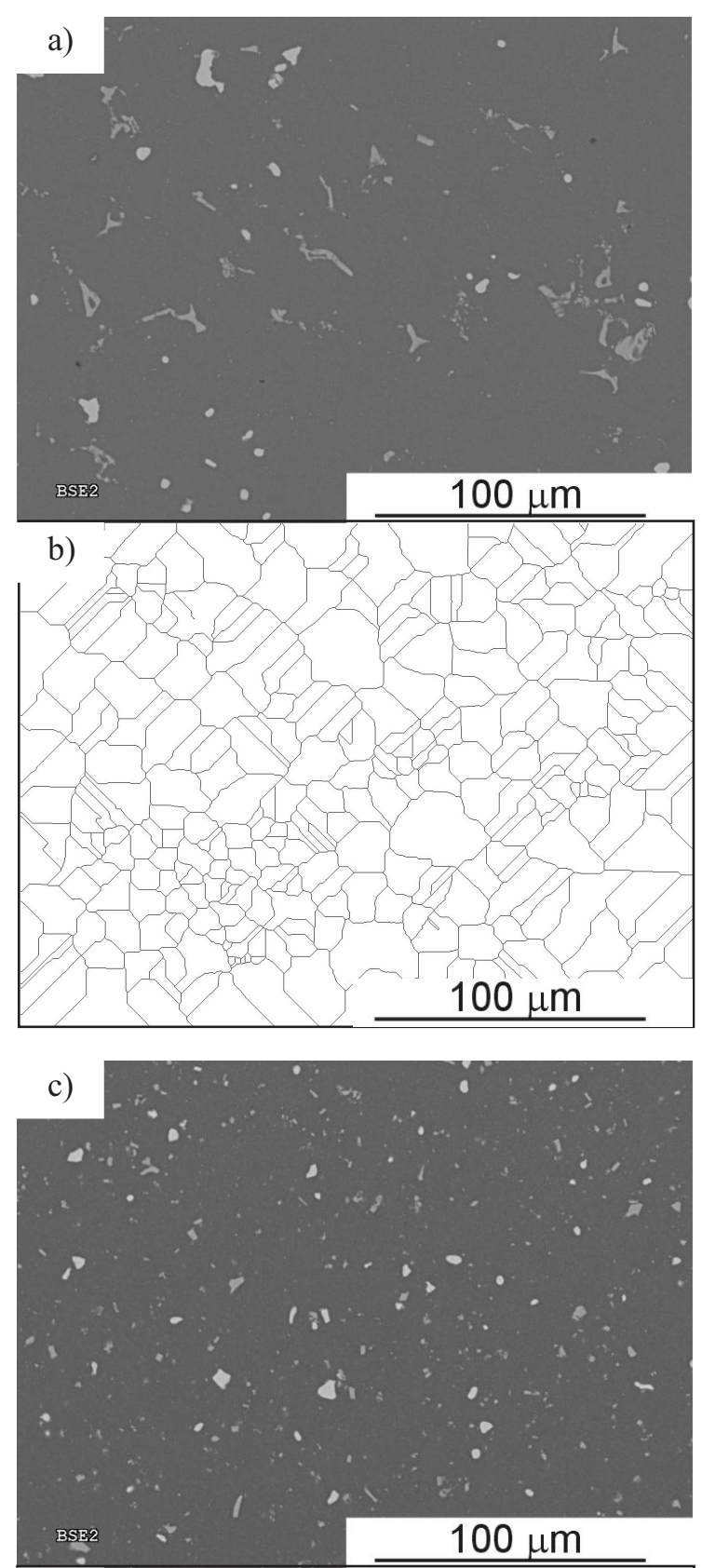

d)

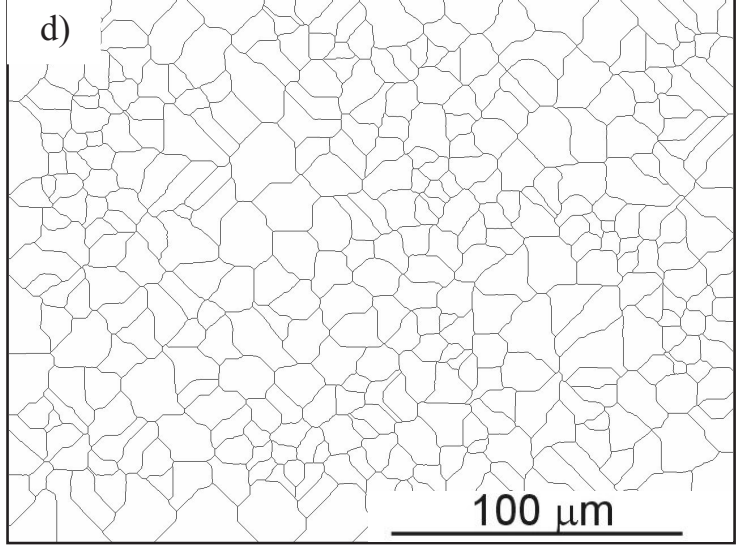

Fig. 19. SEM micrographs and related Voronoi cells for nano-particles in 2017 aluminium alloy before $(a, b)$ and after $(c, d)$ hydrostatic extrusion.
An example of quantitative description of particle arrangements is presented in Fig. 19, which explains the steps executed in the application of SKIZ tessellation method. In this method, each particle is surrounded with a cell, which size depends on its distance to the neighbouring particles. The particles imaged in Fig. 19 are intermetallic inclusions in aluminium alloy. As can be seen, the spatial arrangement of these inclusions changes with the plastic deformation via hydrostatic extrusion. Similar changes are observed in the case of "plastic deformation" by mechanical mixing of nano-silica particles dispersed in a polymeric matrix.

\section{CONCLUSIONS}

The following three conclusions seem to be a good summary of the considerations described in the present paper.

First, there has been a tremendous drive towards development of nano-engineering materials. In particular an impressive progress has been made in the improvement of nano-powders and nanoparticulate systems. One should also acknowledge remarkable progress in fabrication of bulk nano-metals via methods of Severe Plastic Deformation.

Second, the progress made in nano-materials was possible due to new techniques for imaging of the nanostructures in engineering metals becoming widely available. Among others, the accessibility to Atomic Force and Scanning (Transmission) Electron Microscopy must be underlined.

Third, the development in nano-materials opens new possibilities for stereology and quantitative description of the microstructure as new engineering materials must be now controlled/designed with increased precision.

\section{ACKNOWLEDGEMENT}

This work was carried out within a NANOMET Project financed under the European Funds for Regional Development (Contract No. POIG.01.03.0100-015/08). The paper was also supported by Polish Ministry of Science and Higher Education under grant No. 110/COS/2006/02. Support of Dr. M. Andrzejczuk, R. Dobosz is kindly acknowledged.

\section{REFERENCES}

Armstrong RW (1983). The yield and flow stress dependence on polycrystal grain size, Yield, Flow and Fracture of Polycrystals. London: Applied Science Publishers. 
Baddeley AJ, Gundersen HJG, Cruz-Orive LM (1986). Estimation of surface from vertical sections. J Microsc 142:259-76.

Bauhofer W, Kovacs JZ (2009). A review and analysis of electrical percolation in carbon nanotube polymer composites. Compos Sci Technol 69:1486-98.

Cherukuri B, Nedkova TS, Srinivasan R (2005). A comparison of the properties of SPD-processed AA6061 by equal-channel angular pressing, multi-axial compressions/forgings and accumulative roll bonding. Mat Sci Eng A 410-411:394-7.

Dobosz R (2009). PhD Thesis. Warsaw University of Technology.

Hanisch KH, Stoyan D (1981). Stereological estimation of the radial distribution function of centres of spheres. $\mathrm{J}$ Microsc 122:131-41.

Kurzydlowski KJ, Ralph B (1995). Quantitative description of the microstructure of materials. Baton Rouge, USA: CRC Press.

Lewandowska M, Pachla W, Kurzydlowski KJ (2007). Fabrication of high strength nanostructured aluminium alloys by hydrostatic extrusion. Int J Mater Res 98:1727.

Lewandowska M, Kurzydlowski KJ (2008). Recent development in grain refinement by hydrostatic extrusion. J Mater Sci 43:7299-306.

Moszner N, Saltz U (2001). New developments of polymeric dental composite. Prog Polym Sci 26:535-76.

Richert M, Liu Q, Hansen N (1999). Microstructural evolution over a large strain range in aluminium deformed by cyclic-extrusion-compression. Mat Sci Eng A 260:275-83.

Ryszkowska J, Zawadzak E, Lojkowski W, Opalinska A, Kurzydlowski KJ (2007). Structure and properties of polyurethane nanocomposite with zirconium oxide including $\mathrm{Eu}^{3+}$. Mat Sci Eng C 27:994-7.

Siejka-Kulczyk J, Mystkowska J, Lewandowska M, Dąbrowski JR, Kurzydłowski KJ (2009). The influence of nano-silica on the wear resistance of ceramicpolymer composites intended for dental fillings. Sol St Phen 151:135-8.

Ungar T, Ribarik G, Gubicza J, Hanak P (2002). Dislocation structure and crystallite size distribution in plastically deformed metals determined by diffraction peak profile analysis. J Eng Mater Technol 124:2-6.

Valiev RZ, Islamgaliev RK, Alexandrov IV (2000). Bulk nanostructured materials from severe plastic deformation. Prog Mater Sci 45:103-89.

Valiev RZ, Langdon TG (2006). Principles of equalchannel angular pressing as a processing tool for grain refinement. Prog Mater Sci 51:881-981.

Widlicki P (2008). PhD Thesis. Warsaw University of Technology.

Wojnar L, Majorek M (1994). Computer based image analysis. FOTOBIT-DESIGN S.C, 87-91.

Zhilayaev AP, Kim B-K, Nurislamova GV, Baro MD, Szpunar JA, Langdon TG (2002). Orientation imaging microscopy of ultrafine-grained nickel. Scripta Mater 46:575-80. 